\title{
Revisión de la investigación educativa sobre el abandono escolar. Tendencias
}

\section{bibliométricas}

\author{
Review of educational research on drop-out. Bibliometric trends \\ Revisão da pesquisa sobre abandono educacional. Tendências bibliométricas
}

Recibido: 20/01/2021 | Revisado: 20/01/2021 | Acepto: 23/01/2021 | Publicado: 30/01/2021

\author{
Abraham Bernárdez-Gómez \\ ORCID: https://orcid.org/0000-0003-1862-5554 \\ Universidad de Murcia, España \\ E-mail: abraham.bernardez@um.es \\ José Santiago Álvarez-Muñoz \\ ORCID: https://orcid.org/0000-0002-9740-6175 \\ Universidad de Murcia, España \\ E-mail: josesantiago.alvarez@um.es \\ María Luisa Belmonte \\ ORCID: https://orcid.org/0000-0002-1475-3690 \\ Universidad de Murcia, España \\ E-mail: maríaluisa.belmonte@um.es
}

\begin{abstract}
Resumen
La finalidad del presente artículo es realizar un análisis bibliométrico de una de las problemáticas educativas con mayor recurrencia en los sistemas educativos, el abandono escolar. Ello emerge de los numerosos intentos que, tanto la administración como desde la investigación, se han realizado por alcanzar una fórmula que consiga paliar la alta incidencia de este fenómeno. El cual, como se puede observar, ha sido un problema de interés al presentarse en la ya vencida estrategia para Europa Horizonte 2020 y considerarse clave en los Objetivos para el Desarrollo Sostenible 2030. La investigación se llevará a cabo mediante un análisis bibliométrico de las bases de datos con mayor representación en el ámbito educativo: ERIC, Dialnet, Teacher Reference center, Web of Science y Scopus. Después de establecer los descriptores y criterios de inclusión/exclusión, se extrajeron 209 referencias en las que se analizó la temática concreta dentro del abandono educativo, los principales autores en el ámbito de investigación y las principales publicaciones sobre este problema educativo, entre otros.
\end{abstract}

Palabras clave: Abandono escolar; Revisión bibliométrica; ODS 2030; Revisión bibliográfica.

\begin{abstract}
The purpose of this article is to carry out a bibliometric analysis of one of the educational problems with the greatest recurrence in education systems, school drop-out. This emerges from the numerous attempts that, both administration and research, have been made to achieve a formula that manages to alleviate the high incidence of this phenomenon. Which, as can be seen, has been a problem of interest in presenting the already overdue Strategy for Europe Horizon 2020 and being considered key to the 2030 Sustainable Development Goals. The research will be carried out through a bibliometric analysis of the databases with the highest representation in the educational field: ERIC, Dialnet, Teacher Reference Center, Web of Science and Scopus. After establishing the descriptors and criteria of inclusion/exclusion, 210 references were extracted in which the specific topic was analyzed within the educational abandonment, the main authors in the field of research and the main publications on this educational problem, among others.
\end{abstract}

Keywords: Dropout; Bibliometric review; SDG 2030; Bibliographic review.

\section{Resumo}

O objetivo deste artigo é realizar uma análise bibliométrica de um dos problemas educacionais com maior recorrência nos sistemas de ensino, a evasão escolar. Isso emerge das inúmeras tentativas que, tanto a administração quanto a pesquisa, têm sido feitas para alcançar uma fórmula que consiga aliviar a alta incidência desse fenômeno. $\mathrm{O}$ que, como se pode ver, tem sido um problema de interesse em apresentar a já atrasada Estratégia para o Horizonte da Europa 2020 e ser considerada chave para os Objetivos de Desenvolvimento Sustentável de 2030. A pesquisa será realizada por meio de uma análise bibliométrica das bases de dados com maior representação na área educacional: ERIC, Dialnet, Teacher Reference Center, Web of Science e Scopus. Após o estabelecimento dos descritores e critérios de inclusão/exclusão, foram extraídas 210 referências em que o tema específico foi analisado dentro do abandono educacional, os principais autores no campo da pesquisa e as principais publicações sobre esse problema educacional, entre outros.

Palavras-chave: Evasão escolar; Revisão bibliométrica; ODS 2030; Revisão bibliográfica. 


\section{Introducción}

Una investigación, ya sea en educación u otra materia, es el proceso de aplicación del método científico que nos permite obtener información relevante para entender un hecho o ahondar en el conocimiento de un ámbito (Casimiro \& Araújo, 2020; Sonmez, 2020). El hecho que atendemos en nuestro proceso de investigación se concreta en un objetivo general:

Conocer cuál es la tendencia en investigación sobre la problemática del abandono escolar mediante una revisión bibliométrica.

Como objetivos específicos planteamos:

- Identificar, enumerar y describir las bases de datos que sean susceptibles de aportar resultados a la búsqueda.

- Registrar las fuentes documentales sobre la problemática a la que nos enfrentamos siguiendo las pautas establecidas en este tipo de investigaciones.

- Indagar sobre las principales publicaciones periódicas y autores de este ámbito.

- Destacar las distintas categorías de estudio o líneas de investigación dentro del abandono escolar.

La lucha contra el abandono escolar posee una relevancia especial en nuestro sistema educativo y así se le reconoce al ubicarlo dentro de los Objetivos para el Desarrollo Sostenible 2030. Ésta indica cinco áreas prioritarias de actuación, que a su vez engloban una serie de objetivos. En el caso del apartado referente a Educación, podemos apreciar que dichos objetivos van de la mano. Estos son: tasas de abandono escolar prematuro por debajo del 10\%, y al menos un $40 \%$ de las personas de 30 a 34 años de edad deberán completar estudios de nivel terciario. Esta demanda responde a la llamada de atención a las administraciones educativas sobre el empobrecimiento formativo de un considerable sector de la población y la consecuente vulnerabilidad en su desarrollo socio-personal.

De su relevancia da buena cuenta también el hecho de que este problema educativo aparece descrito como un asunto sobre el que intervenir con carácter de urgencia en diversos textos anteriores (Belmonte \& Bernárdez-Gómez, 2020; Bernárdez-Gómez \& Belmonte, 2020a, 2020b; Hernández \& Álvarez, 2019). Dónde se indica la necesidad de mejorar esta situación de los alumnos de educación media debido a las consecuencias generadas para su desarrollo y por ser un factor de exclusión socioeducativa que no garantiza la existencia de la equidad en la educación media.

Ésta última, definida en la LOMCE como "la igualdad de oportunidades para el pleno desarrollo de la personalidad a través de la educación, la inclusión educativa, la igualdad de derechos y oportunidades que ayuden a superar cualquier discriminación y la accesibilidad universal a la educación, y que actúe como elemento compensador de las desigualdades personales, culturales, económicas y sociales" no hace más que recordarnos a la idea de centros amables en los que, "como resultado de la colaboración de todos" se producen mejores condiciones para que la enseñanza se desarrolle ofreciendo las mejores oportunidades para que los alumnos se desarrollen (Trillo, Parada \& Bernárdez-Gómez, 2020).

Por último, para dar respuesta a los objetivos planteados, se ha estructurado el presente trabajo en los siguientes tres apartados:

1. En un primer lugar podremos introducirnos en el marco de investigación del trabajo. Dado que se trata de una revisión bibliométrica, expondremos una serie de premisas metodológicas a tener en cuenta en la realización de una investigación de estas características (Patiño Toro et al., 2020; Phillips \& Ozogul, 2020; Samul, 2020; Shareefa \& Moosa, 2020; Sökmen \& Nalçaci, 2020).

2. Uno segundo, en el que se describe el proceso de revisión, en el que daremos cuenta de las tareas llevadas a cabo para localizar, seleccionar y gestionar las fuentes bibliográficas que surjan como resultado de la búsqueda (Gümüs et al., 2020; Hao et al., 2020; Hernández et al., 2017; Ivanovic \& Ho, 2019; Lei \& Liu, 2019; Velasco et al., 2020).

3. Un tercero en el que presentamos el análisis de la información encontrada, concretando aspectos referentes a las publicaciones, autores relevantes en el campo de estudio y las líneas de investigación que se están trabajando en la actualidad 
(do Nascimento et al., 2019; Julia et al., 2020; Liu et al., 2020; Song \& Wang, 2020).

\section{Método}

Los documentos y textos escritos son la principal herramienta y medio de transmisión del conocimiento desde hace siglos, constituyendo un patrimonio cultural y científico sin parangón. En la actualidad, la digitalización de estos ha supuesto un acercamiento a la sociedad y ha facilitado la propagación los distintos saberes reflejados en ellos. En consecuencia, la utilización de estos materiales es esencial para toda investigación científica. Así pues, lo que se presenta en estas hojas es un informe de revisión bibliográfica sobre el objeto de estudio marcado: el abandono escolar.

Este método posibilitará realizar una economía de esfuerzo dado que se concentrará la atención en los textos seleccionados de forma más eficiente, depurando los elementos esenciales de los accesorios. De esta forma, conseguimos equilibrar nuestro conocimiento de una forma objetiva mediante la aplicación del método científico (Chaves \& Puentes, 2020; Martínez et al., 2020; Pacetti-Donelson, 2018; Rodríguez Jiménez et al., 2019). Así, realizamos una investigación secundaria limitada a recabar y analizar datos de otros investigadores, estableciéndose como etapa preliminar esencial en cualquier investigación empírica o, como es el presente caso (Julia et al., 2020; Montero et al., 2020; Özkaya, 2018; Ramírez et al., 2020), siendo investigación independiente, proporcionando, en ambos, una síntesis del conocimiento acerca de un tema específico. Además, este tipo de investigaciones contribuyen a familiarizarse con el tema de estudio y estructurar las ideas que dieron origen al mismo.

Para la realización de este proceso se proponen distintas etapas de trabajo con distinto dinamismo y complejidad para el investigador (Carlsson et al., 2017; Esen et al., 2020; Hernández-Prados et al., 2019; Ramírez et al., 2020). Se ha elegido una propuesta que sistematizó las distintas fases de búsqueda de información que realizó el grupo de personas que constituía su grupo de análisis (Aksu \& Güzellerii, 2019; Asmat Vega et al., 2019; Robles et al., 2020; Saz, 2020). Estas son:

- Inicio, reconociendo una necesidad de información.

- Selección, centrándose en escoger el enfoque y los aspectos que se van a seguir.

- Exploración, con una investigación general, con vista a incrementar la comprensión.

- Formulación, estableciendo la hipótesis final de trabajo y así reducir la incertidumbre.

- Colección, localizando y seleccionando la información relacionada con el tema.

- Acción, empleando el nuevo conocimiento adquirido para el fin planteado.

Con ello, pretendemos dar cuenta de cómo ha sido el proceso y tratar de realizar una tarea lo más sistematizada y rigurosa posible, que explicamos en el apartado referente a la metodología. A continuación, exponemos brevemente como se han escogido las distintas bases de datos para realizar las búsquedas y daremos unas pinceladas sobre cada una de ellas.

\subsection{Las bases de datos en educación}

"Se trata de una fuente muy utilizada en el ámbito de las bibliotecas de investigación y académicas, y sirve para saber qué se ha publicado en revistas, actas de congresos, tesis, tesinas, libros o capítulos de libros de una especialidad concreta. Es, por tanto, un recurso que las bibliotecas y los centros de documentación adquieren para que los usuarios sepan qué se ha publicado." (Somoza, 2015 p. 13)

Como cualquier investigación, nuestro proceso también ha estado supeditado a los recursos disponibles. De esta forma, identificamos las principales bases de datos en el ámbito de la Educación en función de los recursos que nos facilite la Universidad de Santiago de Compostela. Ésta da acceso a una herramienta de localización de recursos denominada "Fiador", 
común a las tres universidades gallegas, de la que hemos extraído las bases de datos más representativas del ámbito educativo.

ERIC: base de datos bibliográfica del Educational Resources Information Center (ERIC). Reúne dos archivos: Resources in Education (RIE) y Current Index to Journals in Education (CIJE), que contiene las referencias a los artículos de revista aparecidos en más de 750 publicaciones periódicas especializadas con más de 700.000 citas, resúmenes y en algunos casos el texto completo de artículos de revistas, ponencias de congresos, informes técnicos, programas educativos y otros documentos de literatura gris. Es un sistema de información patrocinado por el Departamento de Educación de los Estados Unidos, que produce la base de datos bibliográfica nacional. Engloba un amplio campo de materias: educación superior, educación elemental, idiomas, recursos de información, lectura, educación rural, matemáticas, ciencias do medio natural, estudios sociales, educación do profesorado. También as posible consultar sólo el ISAB Subset, subconjunto de registros incluidos en la base ERIC, que se refieren a temas de ciencias de la información en relación con la educación. Contiene datos desde 1966 y se actualiza mensualmente.

DIALNET: es una base de datos de acceso libre fruto de un proyecto cooperativo entre diversas universidades españolas y liderado por la Universidad de La Rioja. Una de las mayores bases de datos de contenidos científicos en lenguas iberoamericanas que integra el mayor número posible de recursos, buscando en la medida de lo posible el acceso a los textos completos de los mismos, apostando claramente por el acceso abierto a la literatura científica. Recoge los índices de gran parte de las revistas científicas y humanísticas de España y Latinoamérica, incluyendo también libros, tesis doctorales y otros tipos de documentos. Además, da acceso a numerosos contenidos a texto completo.

Ofrece a investigadores, y usuarios en general, diversos servicios personalizados y gratuitos, por ejemplo, la subscrición de alertas informativas. Para hacer uso de estos servicios es necesario estar registrado en el sistema. Además posee una página personalizada para las instituciones colaboradoras, favoreciendo así la visibilidad y accesibilidad a los contenidos de estas instituciones.

Teacher Reference Center: Contiene índices y resúmenes de revistas, publicaciones periódicas y monografías en el área de la docencia. Abarca temas como la evaluación, educación permanente, investigación pedagógica, desarrollo del currículo o métodos para la enseñanza.

Web of Science: plataforma web (anteriormente llamada Web of Knolewdge) que da acceso a recursos multidisciplinares con información bibliográfica de trabajos publicados en las revistas científicas más prestigiosas, así como herramientas de análisis de las propias publicaciones. Dentro de ella nos encontramos con:

WEB of SCIENCE (WoS), índice de citas de cerca de 9.300 publicaciones desde el año 1900. Se divide en Science Citation Index Expanded, Social Sciences Citation Index (desde 1956-, ofrece información sobre citas e información bibliográfica referencial en el campo de las ciencias sociales, de unas 1.810 revistas y 30 libros más citados. Actualización diaria) y Arts \& Humanities Citation Index.

- JOURNAL CITATION REPORTS (JCR), herramienta de evaluación de más de 7.600 publicaciones revisadas por pares y más citadas del mundo, de aproximadamente 220 disciplinas de más de 3.300 editores (desde 1997- ).

- $\quad$ ESSENCIAL SCIENCE INDICATORS, datos de investigación y estadísticas que miden el rendimiento y las tendencias en investigación en los últimos 10 años.

SCOPUS: es una base de datos de referencias bibliográficas, resúmenes y citas del grupo editorial Elsevier. Recoge información de diferentes publicaciones (revistas científicas, revistas especializadas, series de libros, conferencias, etc.) de las diferentes áreas del conocimiento. Contiene referencias bibliográficas de 16.300 publicaciones periódicas (peer-review) procedentes de 4.000 editoriales, 700 actas de congresos internacionales, 1.200 revistas openacces, 386 millones de páginas científicas, 22 millones de patentes y las bases de datos MEDLINE y EMBASE. Actualmente se encuentra integrado en la plataforma SciVerse Scopus, junto con Science Direct y el contenido web de SCIRUS (buscador científico de internet). 
Proporciona referencias bibliográficas completas, más de 29 millones de resúmenes y permite buscar por referencias citadas desde el año 1996 en adelante.

\section{Proceso de revisión}

Una vez señaladas las bases de datos en las que realizar la búsqueda documental se puede dar paso a explicar cómo ha sido el camino seguido para la revisión y los resultados que ésta ha arrojado. Conocidas las bases de datos, presentamos ahora el procedimiento seguido para identificar, definir y sistematizar la información que nos ofrecen.

Comenzamos por acceder a "FIADOR", la herramienta de recursos electrónicos creada por el consorcio de bibliotecas universitarias de Galicia, accesible en http://sp.bugalicia.org/.

Al seleccionar las bases de datos, distinguimos los recursos por cada área de conocimiento. Una vez indagamos en la web, advertimos que si trabajamos sobre la materia específica de educación nuestra búsqueda se vería mermada, dado que se entiende la existencia de bases de datos generales para el área de ciencias sociales.

Una vez hecho esto, necesitamos identificar los distintos tesauros establecidos por Unesco que darían lugar a los descriptores a emplear en la búsqueda. Accedemos a la base de datos ERIC, contenedora de dicha lista de tesauros. Dado que se trata de una base de datos en inglés, hicimos la búsqueda en dicha lengua, estableciendo que el término estandarizado en el catálogo de la UNESCO es “dropouts” (Ver imagen 1).

Entre los numerosos tesauros que aparecen (ver imagen 2), los que mejor se integran en nuestro objeto de estudio y los establecidos como líneas de investigación referentes en la literatura son los 5 siguientes: Dropout Attitudes, Dropout Characteristics, Dropout Prevention, Dropout Programs y Dropout Research. Por lo que descartamos los no señalados en la imagen 2. Podemos observar que dichos tesauros se establecen en una lengua concreta, por lo que debemos realizar las adaptaciones oportunas según las necesidades encontradas.

Una vez especificados los descriptores de búsqueda que vamos a utilizar, seleccionaremos las bases de datos que, como señalamos con anterioridad, se extrajeron de "FIADOR". Estas serán las indicadas páginas atrás, ERIC, DIALNET, SCOPUS, Teacher Reference Center (TRC) y Web of Science (WOS). A su vez, estableceremos los distintos criterios de inclusión y exclusión, así como los operadores booleanos que guiaran nuestra revisión. Además, aplicaremos distintos filtros o limitadores en las búsquedas, siendo estos, textos escritos desde el 2010, que estén completos de forma accesible y que sean de la materia objeto de estudio. Sin embargo, en el caso de ser necesario alguno a mayores, se indicará posteriormente.

Así pues, en las siguientes páginas se encontrará una lista de referencias que irá aumentando de forma progresiva a medida que se realizan las distintas búsquedas. Señalar que la gestión del material extraído se realizará mediante el programa especializado "Zotero", que facilita la exportación de las referencias desde los navegadores y las organiza según los parámetros que introduzcamos.

\section{Resultados}

En el apartado que se da comienzo en estas líneas, se tratará de dar respuesta a dos de los objetivos planteados al comienzo de este proceso:

-Indagar sobre las principales publicaciones periódicas y autores de este ámbito.

-Destacar las distintas categorías de estudio o líneas de investigación dentro del abandono escolar

Después de establecer un listado manejable de ellas mediante el gestor bibliográfico Zotero y volcar los documentos en el programa de análisis cualitativo ATLAS.Ti, procedimos a elaborar un informe que responda a estos objetivos.

Como podemos observar en la Tabla 1, en el resultado final de la búsqueda hemos obtenido 48 referencias en la base de datos ERIC, 46 en Dialnet, 14 en TRC, 41 en WOS y 61 en SCOPUS, siendo un total de 209 citas bibliográficas las 
encontradas. De estos datos podemos destacar la diferencia existente entre la base de datos TRC y el resto de ellas, dado que en la primera se encontró un número escaso de referencias. Además, también cabe destacar la Base de datos SCUPUS, puesto que en ella aparecen mayor número de referencias.

Tabla 1. Número de referencias por base de datos y descriptor.

\begin{tabular}{lccccc}
\hline & ERIC & DIALNET & TRC & WOS & SCOPUS \\
\hline Dropout Attitudes & 8 & & 0 & 3 & 8 \\
Dropout Characteristics & 14 & & 4 & 10 & 18 \\
Dropout Prevention & 9 & & 3 & 10 & 11 \\
Dropout Programs & 11 & & 4 & 8 & 12 \\
Dropout Research & 6 & & 3 & 10 & 12 \\
Abandono escolar Y programa & & 5 & & & \\
Abandono escolar Y factores & & 12 & & & \\
Abandono escolar E investigación & & 10 & & & \\
Abandono escolar & & 19 & & & \\
Total & 48 & 46 & 14 & 41 & 61 \\
\hline
\end{tabular}

Fonte: Autores.

\subsection{Revistas científicas}

Ya hemos indicado anteriormente los objetivos que se pretenden en el presente punto. También se han precisado las 209 fuentes encontradas en las distintas bases de datos en relación a la problemática que rodea el abandono escolar. Una vez hecho esto, a continuación veremos en la Tabla 2 una enumeración de las distintas publicaciones que poseen más de una referencia de nuestra búsqueda. Esto lo hemos realizado con la opción que facilita Zotero para ordenar las citas por nombre de publicación y de mayor a menor cantidad de citas. Además, se indicará el cuartil en el que se ubica dicha revista dentro del índice de referencia para publicaciones de la editorial Thomsom Reuters. 
Tabla 2. Número de referencias por revista.

\begin{tabular}{|c|c|c|}
\hline Nombre de la revista & Ref. & Indexación JCR \\
\hline 1. Revista de educación & 14 & Q3 \\
\hline 2. European Journal of Education & 9 & Q3 \\
\hline $\begin{array}{l}\text { 3. Avances en supervisión educativa: Revista de la Asociación de } \\
\text { Inspectores de Educación de España }\end{array}$ & 6 & \\
\hline 4. British Journal of Educational Psychology & 4 & Q2 \\
\hline 5. Education and Urban Society & 4 & Q4 \\
\hline 6. Journal of Educational Research & 4 & Q2 \\
\hline 7. Zeitschrift Fur Padagogik & 3 & Q4 \\
\hline 8. Teachers College Record & 3 & Q3 \\
\hline $\begin{array}{l}\text { 9. School Dropout and Completion: International Comparative Studies in } \\
\text { Theory and Policy }\end{array}$ & 3 & \\
\hline 10. Revista de la Asociación de Sociología de la Educación (RASE) & 3 & \\
\hline 11. Research in Higher Education & 3 & Q2 \\
\hline $\begin{array}{l}\text { 12. REICE: Revista Electrónica Iberoamericana sobre Calidad, Eficacia y } \\
\text { Cambio en Educación }\end{array}$ & 3 & \\
\hline 13. Praxis sociológica & 3 & \\
\hline 14. Journal of School Counseling & 3 & \\
\hline 15. European Journal of Psychology of Education & 3 & Q3 \\
\hline 16. Educational Review & 3 & Q3 \\
\hline 17. Economics of Education Review & 3 & Q2 \\
\hline 18. ACLPPinforma & 2 & \\
\hline 19. Educational Evaluation and Policy Analysis & 2 & Q1 \\
\hline 20. Enseñanza \& Teaching: Revista interuniversitaria de didáctica & 2 & \\
\hline $\begin{array}{l}\text { 21. International Review of Education / Internationale Zeitschrift für } \\
\text { Erziehungswissenschaft }\end{array}$ & 2 & \\
\hline 22. Journal of Education for Students Placed at Risk & 2 & \\
\hline 23. Journal of Youth and Adolescence & 2 & Q1 \\
\hline 24. Kuram Ve Uygulamada Egitim Bilimleri & 2 & Q4 \\
\hline 25. Learning and Individual Differences & 2 & Q2 \\
\hline 26. Phi Delta Kappan & 2 & Q4 \\
\hline 27. Revista Electrónica Educare & 2 & \\
\hline 28. Social Science Research & 2 & Q1 \\
\hline
\end{tabular}

Fonte: Autores.

Como se puede ver en la tabla que precede estas líneas, existen tres publicaciones que destacan frente a las otras: Revista de Educación, European Journal of Education y Avances en supervisión educativa: Revista de la Asociación de Inspectores de Educación de España. En el resto de ellas apreciamos que aparecen escasas publicaciones. En la mayoría de las publicaciones, las citas que se encuentran son de una o dos referencias de nuestra lista. También es destacable que un alto porcentaje de las revistas donde se publica pertenezcan a las denominadas revistas de impacto o JCR, un indicador de calidad 
empleado en la actualidad.

A continuación, lo que se presenta es una indagación un poco más profunda de cada una de las revistas destacadas. Con ello, pretendemos comprender mejor la relevancia de cada una de ellas en nuestra materia de estudio.

Revista de educación: es una publicación científica del Ministerio de Educación, Cultura y Deporte español. Fundada en 1940, y manteniendo el título de Revista de Educación desde 1952, es un testigo privilegiado de la evolución de la educación en las últimas décadas, así como un reconocido medio de difusión de los avances en la investigación y la innovación en este campo, tanto desde una perspectiva nacional como internacional. La revista es editada por la Subdirección General de Documentación y Publicaciones, y actualmente está adscrita al Instituto Nacional de Evaluación Educativa de la Dirección General de Evaluación y Cooperación Territorial.

European Journal of Education: es una revista académica internacional en inglés sobre educación europea, publicada trimestralmente por Wiley-Blackwell para el Instituto Europeo de Educación y Política Social e indexada por Thomson Reuters para adquirir factor de impacto. Cada número está dedicado a un tema en particular. Las contribuciones son encomendadas a una serie de especialistas, con el fin de lograr una perspectiva orientada a políticas europeas. La revista busca activamente asociarse con organizaciones (por ejemplo, universidades, fundaciones, organizaciones internacionales) con miras a organizar seminarios sobre temas temáticos o futuros.

Avances en supervisión educativa: Revista de la Asociación de Inspectores de Educación de España: La revista se dirige, por su vocación interdisciplinar y de divulgación, a los inspectores e inspectoras de educación, a la comunidad educativa, y al público en general, interesados en la realidad educativa nacional e internacional. Esta pretende ser un instrumento de encuentro en el ámbito educativo para el intercambio de información y experiencias en la búsqueda de aunar pensamientos y voluntades con el fin de ir avanzando en propuestas que sean asumidas y consensuadas en aquellos temas educativos, y profesionales, que son de máximo interés.

Tras la lectura e indagación para realizar estas breves reseñas de la historia de las publicaciones, Revista de Educación, European Journal of Education y Avances en supervisión educativa, podemos señalar una serie de factores que confluyen en ambas. Todos ellas son arbitradas exteriormente, con lo que se realiza una primera revisión con una calidad garantizada. Se publica investigación básica y aplicada, por lo que son accesibles a un a la investigación que da sus primeros pasos. Y, por último, ambas poseen una estructura diferenciada para investigación, ensayos, monográficos, etc.

\subsection{Autores de referencia para nuestro objeto de estudio}

Continuando con nuestra andadura, recordamos que uno de nuestros objetivos era señalar los autores relevantes, o que tuviesen investigaciones de referencia, en la búsqueda realizada sobre nuestro ámbito de estudio, la problemática sobre abandono escolar.

Para la realización de esta tarea hemos vuelto a utilizar el programa Zotero, esta vez con la opción de ordenar las publicaciones por nombre de autor. Una vez hecho esto se procedió a examinar qué autores eran los que poseían mayor número de referencias. En dicha lista se encuentra un número de autores muy variado sin ningún grupo de nombres significativos que destacar. Sin embargo, después de explorar los primeros nombres en redes sociales de investigación comenzaron a considerarse tres nombres como los más relevantes de este ámbito en el momento que nos encontramos: Alez Bowers, Kristof De Witte y Laurier Fortin. A continuación, se expone una breve reseña biográfica de los mismos.

Alex Bowers, Profesor Asociado de Liderazgo Educativo de la Universidad de Columbia, donde trabaja para ayudar a los equipos directivos a utilizar los datos que ya recogen en las escuelas de manera más efectiva para ayudar a dirigir los limitados recursos de las escuelas a las necesidades específicas de los estudiantes. Su investigación se centra en la intersección del liderazgo efectivo de la escuela, la organización y los recursos humanos, la toma de decisiones impulsada por los datos, las 
calificaciones de los estudiantes y de los exámenes, la persistencia del estudiante y el abandono escolar.

Kristof De Witte, es profesor titular permanente en la Facultad de Ciencias Económicas y Busin ess en KU Leuven, Bélgica, y tiene la cátedra de "Eficacia y Eficiencia de Innovaciones Educativas) en el Top Institute for Evidence Based Education Investigación (TIER) en la Universidad de Maastricht, Países Bajos. Los intereses de investigación de Kristof De Witte abarcan la economía de la educación, la mejora innovación en evaluación y el abandono escolar prematuro. Es presidente del Comité de Programa del programa post-inicial del MEBIT para profesores y coordina el programa de formación de profesores de secundaria superior.

Laurier Fortin, profesor titular en el departamento de psicología educativa de la Universidad de Sherbrooke, posee una larga carrera en cuanto a investigación sobre abandono escolar se refiere. Como titular de la Cátedra de Investigación de la Junta Escolar de la Región de Sherbrooke, ha llevado a cabo un estudio longitudinal durante 11 años sobre engagement educativo y abandono escolar, realizando una aportación de consideración para el campo de estudio.

Además de estos, también encontramos otros que se pronuncian al desarrollar investigación sobre la problemática de estudio Esto se motiva en función de sus aportaciones al ámbito educativo. Algunos de ellos son:

- Tudorel Andrei: profesor en la Academia de Estudios Económicos de Bucarest, con tres referencias.

- Colin Campbell: profesor en la Universidad de Wisconsin, EE.UU, con dos textos.

- Anna-Maria Fall: profesora en la Universidad de Texas. EE.UU, con dos de ellas.

- Youngju Lee: profesora en la Universidad Nacional de Educación de Korea, que aporta tres textos.

- Anne Lessard: compañera de Fortin, también es profesora en la Universidad de Sherbrooke con dos referencias.

- Aina Tarabini: profesora en la Universidad Autónoma de Barcelona con dos aportaciones al haber científico sobre el abandono escolar.

Finalizada esta parte del análisis cabe decir que ha resultado gratificante encontrar este número de investigaciones y comprobar la profundidad y vinculación de los equipos de investigación en alguna de ellas.

A continuación, daremos paso a uno de los apartados que, a juicio personal, tiene mayor complejidad debido a la necesidad de enlazar los documentos aparecidos en la búsqueda sobre la problemática referente al abandono escolar.

\subsection{Categorías de análisis alrededor de la problemática del abandono escolar}

Como se había señalado al comienzo de este trabajo, uno de nuestros objetivos era: destacar las distintas categorías de estudio o líneas de investigación dentro del abandono escolar. En esta dirección, se ha procedido a analizar mediante el programa de investigación cualitativa ATLAS.Ti las principales líneas sobre las que se estructuran los distintos textos. Aunque se ha realizado alguna lectura de la bibliografía seleccionada, el análisis se ha realizado en función de los títulos, resúmenes y palabras clave, por lo que será susceptible de modificación cuando se proceda a la lectura completa del texto.

De esta forma, se pretende realizar un mapa que señale las distintas líneas de trabajo y los vínculos existentes entre los distintos ámbitos, ya que suponemos un apoyo entre ellas. Como podemos ver a través de la Figura 1, hemos hallado un total de 11 ejes en torno a los que gira la problemática referente al abandono escolar, a las que daremos explicación en las siguientes líneas. 
Figura 1. Fundamentación de líneas de investigación.

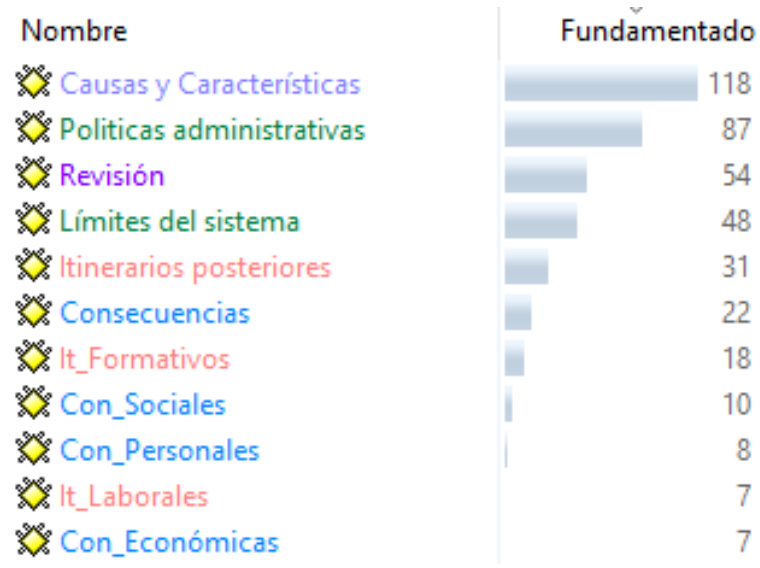

Fonte: Autores.

\section{Discusión y conclusiones}

El objetivo de este trabajo era conocer cuál es la tendencia en investigación sobre la problemática del abandono escolar mediante una investigación bibliométrica. La dirección marcada por dicho objetivo se articulaba en una serie de objetivos específicos que no servían para manejar mejor la consecución del primero. Así pues, cabe dar cuenta de los resultados en cada uno de ellos.

El primero de ellos invitaba a comenzar el proceso mediante la identificación, enumeración y descripción de las bases de datos que sean susceptibles de aportar resultados a nuestra búsqueda. Gracias a la aplicación "fiador" de la red de bibliotecas universitarias de Galicia identificábamos cinco de ellas y realizábamos sus correspondientes descripciones (ERIC, Dialnet, Scopus, TRC y WOS). Como se pudo apreciar, dichas bases nos aportaban distintos tipos de variables y operadores para realizar la búsqueda y ajustarla a nuestras necesidades, siendo más eficientes en el proceso, además de poder sistematizarlo.

El siguiente objetivo era registrar las fuentes documentales sobre la problemática a investigar siguiendo las pautas establecidas en este tipo de investigaciones. Esto se vio facilitado por las herramientas informáticas existentes en la actualidad. Gracias al programa de gestión bibliográfica Zotero, podíamos capturar las referencias a golpe de clic a la vez que nos brindaba la posibilidad de manipularlas en base a los criterios que se necesitarían con posterioridad. Además, dicho programa se puede configurar para las distintas normas de citación existentes en la actualidad como la Cell, Chicago, APA, Elsevier, IEEE, Nature, Vancouver, etc.

De esta manera, también se ha dado respuesta al tercer objetivo planteado, indagar sobre las principales publicaciones periódicas y autores de este ámbito. Con ayuda del programa de gestión se han identificado las principales revistas científicas que poseen un mayor número de publicaciones en este ámbito. A su vez también facilitaba el manejo de los datos en cuanto a los distintos autores que suscriben dicho haber científico. Esta labor ha facilitado la creación de estrategias y habilidades para desarrollar búsquedas precisas y eficaces, como la complementación de la información obtenida en las bases de datos con otra procedente de distintas fuentes.

A modo de último objetivo se destacaron las distintas categorías de estudio o líneas de investigación dentro del abandono escolar. Siendo capaces de extraer las distintas temáticas que giran en torno a la problemática del abandono escolar con la mediación de otro programa informático, el ATLAS.Ti, de análisis cualitativo. Así, entre otras, se señalaban cómo las 
principales investigaciones se centraban en: el funcionamiento de programas de prevención y como se desarrolla su implantación; las características de los alumnos que abandonan sus estudios y cuáles son las causas que llevan a esta situación; las consecuencias, tanto económicas, sociales o personales, que se derivan de esta suceso; los itinerarios posteriores que bu scan corregir, bien las consecuencias, o bien la integración socio-laboral de dicho alumnado; y, por último, las distintas revisiones que se hacen sobre la problemática.

\section{Agradecimientos}

Ayudas a contratos predoctorales FPI. Ref: BES-2017-081040.

\section{Referencias}

Aksu, G., \& Güzellerii, C. O. (2019). Analysis of Scientific Studies on Item Response Theory by Bibliometric Analysis Method. International Journal of Progressive Education, 15(2), 44-64.

Asmat Vega, N. S., Borja Villanueva, C. A., Bernuy Torres, L. A., Lizarzaburu Aguinaga, D. A., \& Morillo Flores, J. (2019). Bibliometric Study of Scientific Production on ICT in Peru (2010-2017). Journal of Educational Psychology - Propositos y Representaciones, 7(2), $203-209$.

Belmonte, M. L., \& Bernárdez-Gómez, A. (2020). Respuesta social al estado de aislamiento por coronavirus, percepciones sobre educación. Revista Conhecimento Online, 3, 30-49. https://doi.org/10.25112/rco.v3i0.2326

Bernárdez-Gómez, A., \& Belmonte, M. L. (2020a). School dropout, determinants, educational policies and subsequent itineraries. Research, Society and Development, 9 (10), p. e6849109234. 10.33448/rsd-v9i10.9234.

Bernárdez-Gómez, A., \& Belmonte, M. L. (2020b). Vulnerabilidad y exclusión educativa, una problemática postergada. Revista GETS, 3 (1), 79-94.

Carlsson, H., Larsson, S., Svensson, L., \& Åström, F. (2017). Consumer Credit Behavior in the Digital Context: A Bibliometric Analysis and Literature Review. Journal of Financial Counseling and Planning, 28(1), 76-94.

Casimiro, A. H. T., \& Araújo, W. J. de. (2020). Cenários prospectivos: Revisão sistemática na Lisa, Emerald, Scopus e Web of Science. Revista Digital de Biblioteconomia e Ciência da Informação, $18(1), 3$.

Chaves, S. R. N., \& Puentes, M. C. S. (2020). Análisis bibliométrico de los efectos veblen, bandwagon, snob, hedónico y de perfeccionismo. CienciAmérica: Revista de divulgación científica de la Universidad Tecnológica Indoamérica, 9(1), 115-137.

do Nascimento, A. S., de Oliveira, F. S., \& Bianconi, M. L. (2019). Bibliometric Analysis of the Brazilian Periodical «Journal of Biochemistry Education». Biochemistry and Molecular Biology Education, 47(3), 249-256. https://doi.org/10.1002/bmb.21220

Esen, M., Bellibas, M. S., \& Gumus, S. (2020). The Evolution of Leadership Research in Higher Education for Two Decades (1995-2014): A Bibliometric and Content Analysis. International Journal of Leadership in Education, 23(3), 259-273. https://doi.org/10.1080/13603124.2018.1508753

Gümüs, H., Gençoglu, C., \& Sahin, T. (2020). Physical Education and Sports: Bibliometric Analysis of the ERIC Database. International Online Journal of Education and Teaching, 7(4), 1823-1837.

Hao, T., Chen, X., \& Song, Y. (2020). A Topic-Based Bibliometric Analysis of Two Decades of Research on the Application of Technology in Classroom Dialogue. Journal of Educational Computing Research, 58(7), 1311-1341. https://doi.org/10.1177/0735633120940956

Hernández Prados, M. Á., \& Álvarez Muñoz, J. S. (2019). Family leisure and academic achievement. Perception of the families. Italian journal of educational research, (23), 86-105. https://ojs.pensamultimedia.it/index.php/sird/article/view/3685

Hernández, J. B., Chalela, S., Arias, J. V., \& Arias, A. V. (2017). Research Trends in the Study of ICT Based Learning Communities: A Bibliometric Analysis. EURASIA Journal of Mathematics, Science \& Technology Education, 13(5), 1539-1562.

Hernández-Prados, M.-Á., García-Sanz, M.-P., Parra, J., \& Gomariz, M.-Á. (2019). Perfiles de participación familiar en Educación Secundaria Obligatoria. Anales de Psicología, 35(1), 84-94. https://doi.org/10.6018/analesps.35.1.325981

Ivanovic, L., \& Ho, Y.-S. (2019). Highly Cited Articles in the Education and Educational Research Category in the Social Science Citation Index: A Bibliometric Analysis. Educational Review, 71(3), 277-286. https://doi.org/10.1080/00131911.2017.1415297

Julia, J., Afrianti, N., Soomro, K. A., Supriyadi, T., Dolifah, D., Isrokatun, I., Erhamwilda, E., \& Ningrum, D. (2020). Flipped Classroom Educational Model (2010-2019): A Bibliometric Study. European Journal of Educational Research, 9(4), 1377-1392.

Julia, J., Supriatna, E., Isrokatun, I., Aisyah, I., Hakim, A., \& Odebode, A. A. (2020). Moral Education (2010-2019): A Bibliometric Study (Part 2). En Online Submission, 8(7), 2954-2968. https://eric.ed.gov/?q=Bibliometric+analysis\&pg=2\&id=ED606763

Lei, L., \& Liu, D. (2019). Research Trends in Applied Linguistics from 2005 to 2016: A Bibliometric Analysis and Its Implications. Applied Linguistics, 40(3), 540-561. https://doi.org/10.1093/applin/amy003 
Liu, Z., Moon, J., Kim, B., \& Dai, C.-P. (2020). Integrating Adaptivity in Educational Games: A Combined Bibliometric Analysis and Meta-Analysis Review. Educational Technology Research and Development, 68(4), 1931-1959. https://doi.org/10.1007/s11423-020-09791-4

Martínez, T. S., Reche, M. P. C., Rodríguez, J. M. R., \& Navas-Parejo, M. R. (2020). Estudio Bibliométrico de los documentos indexados en Scopus sobre la Formación del Profesorado en TIC que se relacionan con la Calidad Educativa. Revista electrónica interuniversitaria de formación del profesorado, 23(2 (Abril)), 19-35.

Montero, F. J. P., Peregrina, C. Á., Tena, M. Á. S., Cruz, F. H. S., \& Collar, C. V. (2020). Análisis bibliométrico de la producción científica en contactología (1936-2019). Gaceta de optometría y óptica oftálmica, 557, 42-47.

Özkaya, A. (2018). Bibliometric Analysis of the Studies in the Field of Mathematics Education. Educational Research and Reviews, 13(22), 723-734.

Pacetti-Donelson, V. (2018). A Bibliometric Analysis of the Proceedings of the Association for Educational Communications and Technology (AECT) for the 1979-2009 Period. En ProQuest LLC. ProQuest LLC.

Patiño Toro, O. N., Acevedo Correa, Y., Valencia-Arias, A., \& Benjumea-Arias, M. (2020). A Bibliometric Analysis of the Use of Open Source Software in Educational Contexts. Problems of Education in the 21st Century, 78(1), 114-128.

Phillips, T., \& Ozogul, G. (2020). Learning Analytics Research in Relation to Educational Technology: Capturing Learning Analytics Contributions with Bibliometric Analysis. TechTrends: Linking Research and Practice to Improve Learning, 64(6), 878-886. https://doi.org/10.1007/s11528-020-00519-y

Ramírez, L. J. C., Roldán, L. S., \& Fernández, G. A. M. (2020). Bibliometría y altmetricas: Una aplicación para estudiantes de doctorado del campo de la educación. XXX Jornadas Luso-Espanholas de Gestão Científica: cooperação transfronteiriça: desenvolvimento e coesão territorial: livro de resumos, 2020, ISBN 978-972-745-273-6, pág. 222, 222. https://dialnet.unirioja.es/servlet/articulo?codigo=7405181

Ramírez, L. J. C., Sánchez-Cañizares, S., \& García, F. J. F. (2020). From Bibliometrics to Entrepreneurship: A Study of Studies. Revista Española de Documentación Científica, 43(3), 2.

Robles, A. S., González, M. E. P., \& Vigil, M. Á. G. (2020). Bibliometric and collaborative network analysis on active methodologies in education. NAER: Journal of New Approaches in Educational Research, 9(2), 259-274.

Rodríguez Jiménez, C., Sanz Prieto, M., \& Alonso García, S. (2019). Technology and Higher Education: A Bibliometric Analysis. Education Sciences, 9. https://eric.ed.gov/?q=Bibliometric+analysis\&pg=2\&id=EJ1231002

Samul, J. (2020). The Research Topics of Leadership: Bibliometric Analysis from 1923 to 2019. International Journal of Educational Leadership and Management, 8(2), 116-143.

Saz, J. T. (2020). Temas y métodos de investigación en Ciencia de la Información, 2000-2019. Revisión bibliográfica. El profesional de la información, 29(4), 19.

Shareefa, M., \& Moosa, V. (2020). The Most-Cited Educational Research Publications on Differentiated Instruction: A Bibliometric Analysis. European Journal of Educational Research, 9(1), 331-349.

Sökmen, Y., \& Nalçaci, A. (2020). A Bibliometric Analysis of the Articles about Values Education. International Journal of Curriculum and Instruction, 12(2), 720-735.

Song, P., \& Wang, X. (2020). A Bibliometric Analysis of Worldwide Educational Artificial Intelligence Research Development in Recent Twenty Years. Asia Pacific Education Review, 21(3), 473-486. https://doi.org/10.1007/s12564-020-09640-2

Sonmez, Ö. F. (2020). Bibliometric Analysis of Educational Research Articles Published in the Field of Social Study Education Based on Web of Science Database. Participatory Educational Research, 7(2), 216-229.

Trillo, F; Parada, A., \& Bernárdez-Gómez, A. (2020). El impacto formativo de la enseñanza en la universidad: una experiencia de autoevaluación docente. Brazilian Journal of Development, 6(9). https://doi.org/10.34117/bjdv6n9-279

Velasco, N. Y. G., Rodríguez, O. Y. A., \& Vargas, A. F. B. (2020). Historia y memoria: Casi 10 años consolidando comunidad historiográfica. Una mirada desde la Bibliometría. Historia y Memoria, 20, 209-247. 